\title{
Management Of Patients With Stroke In Critical Care Units, Considering Osmotic Therapy And Hypothermia
}

Ata Mahmoodpoor

Associate Professor of Anesthesiology, Fellowship of Critical Care Medicine, Faculty of Medicine, Tabriz University of Medical Sciences, Iran.

*Corresponding Author: Email: amahmoodpoor@yahoo.com

Cytotoxic brain edema is an early complication of stroke which increases the possibility of secondary ischemia. Hypertonic solutions, mannitol and recently hypertonic saline (HS) has been considered for treatment of increased ICP. HS could decrease ICP especially in hypotensive patients with different mechanisms, direct effect on edema, decreasing inflammation which is mediated by attenuation of TNFa and IL-1b stimulation on Na-K-Cl cotransporter 1 and improvement of microcirculation. Improvement of microcirculation is so important for hypertonic solutions to be effective in ischemia especially focal ischemia. Based on the literature, hypertonic saline is more effective in decreasing cerebral edema than the equal volume of mannitol. The optimal dose and duration of therapy needs more trials. Caution should be performed with patients with moderate size hemispheric infarcts on presentation, race and genetic factors regarding osmotic therapy.

Hypothermia has been rated as one of the most active modes of neuroprotection based on the results of different trials. Hypothermia in both ways, surface and intravascular, decreases cerebral metabolic rate of 02 and glucose and reduces brain oxygen consumption, inflammation and oxidative stress. Recent data continue to support consideration of therapeutic hypothermia for cerebral ischemia in larger clinical trials of acute ischemic stroke. By increasing the time window to therapy initiation and decreasing the treatment duration, selective intracarotid cold saline administration brings increased feasibility, potentially better outcomes and perhaps fewer complications compared with the whole body cooling. Hypothermia is now recommended as a targeted temperature management with defined protocol which should be started early; it may be performed pharmacologically in combination with other therapies. Applying hypothermia should be considered regarding its cost, using in awaked patients, re-warming protocol, incorporation of thrombolysis and its complications.

Key words: osmotic therapy, hypothermia

DOI: $10.7575 /$ aiac.abcmed.ca1.58

Published Date: February 2017

Peer-review is under responsibility of the 9th Iranian Stroke Congress.

Published by Australian International Academic Centre, Australia

This published work is open access under the CC BY license.

Available online at www.abcmed.aiac.org.au 